\title{
BMJ Open Music to prevent deliriUm during neuroSurgerY (MUSYC) Clinical trial: a study protocol for a randomised controlled trial
}

\author{
Pablo R Kappen (D) , ${ }^{1}$ Johannes Jeekel, ${ }^{2,3}$ Clemens M F Dirven, ${ }^{1}$ M Klimek (D) , ${ }^{4}$ \\ Steven A Kushner, ${ }^{5}$ Robert-Jan Osse, ${ }^{5}$ Michiel Coesmans, ${ }^{5}$ Marten J Poley, ${ }^{6,7}$ \\ Arnaud J P E Vincent ${ }^{1}$
}

To cite: Kappen PR, Jeekel J, Dirven CMF, et al. Music to prevent deliriUm during neuroSurgerY (MUSYC) Clinical trial: a study protocol for a randomised controlled trial. BMJ Open 2021;11:e048270. doi:10.1136/ bmjopen-2020-048270

- Prepublication history and additional supplemental material for this paper are available online. To view these files, please visit the journal online (http://dx.doi.org/10.1136/ bmjopen-2020-048270).

Received 28 December 2020 Accepted 16 September 2021

Check for updates

(C) Author(s) (or their employer(s)) 2021. Re-use permitted under CC BY-NC. No commercial re-use. See rights and permissions. Published by BMJ.

For numbered affiliations see end of article.

Correspondence to

Dr Pablo R Kappen;

p.kappen@erasmusmc.nl

\section{ABSTRACT}

Introduction Delirium is a neurocognitive disorder characterised by an acute and temporary decline of mental status affecting attention, awareness, cognition, language and visuospatial ability. The underlying pathophysiology is driven by neuroinflammation and cellular oxidative stress. Delirium is a serious complication following neurosurgical procedures with a reported incidence varying between $4 \%$ and $44 \%$ and has been associated with increased length of hospital stay, increased amount of reoperations, increased costs and mortality.

Perioperative music has been reported to reduce preoperative anxiety, postoperative pain and opioid usage, and attenuates stress response caused by surgery. We hypothesize that this beneficial effect of music on a combination of delirium eliciting factors might reduce delirium incidence following neurosurgery and subsequently improve clinical outcomes.

Methods This protocol concerns a single-centred prospective randomised controlled trial with 6 months follow-up. All adult patients undergoing a craniotomy at the Erasmus Medical Center in Rotterdam are eligible. The music group will receive recorded music through an overear headphone before, during and after surgery until postoperative day 3. Patients can choose from music playlists, offered based on music importance questionnaires administered at baseline. The control group will receive standard of clinical care

Delirium is assessed by the Delirium Observation Scale and confirmed by a delirium-expert psychiatrist according to the DSM- 5 criteria. Risk factors correlated with the onset of delirium, such as cognitive function at baseline, preoperative anxiety, perioperative medication use, depth of anaesthesia and postoperative pain, and deliriumrelated health outcomes such as length of stay, daily function, quality of life (ie, EQ-5D, EORTC questionnaires), costs and cost-effectiveness are collected.

Ethics and dissemination This study is being conducted in accordance with the Declaration of Helsinki. The Medical Ethics Review Board of Erasmus University Medical Center Rotterdam, The Netherlands, approved this protocol. Results will be disseminated via peer-reviewed scientific journals and conference presentations.

Trial registration numbers NL8503 and NCT04649450.
Strengths and limitations of this study

- This study is the first randomised controlled trial evaluating the effects of recorded music on postoperative delirium in a neurosurgical cohort.

To our knowledge, this is the largest study assessing the effects of music on delirium.

- Both the short-term and longer-term deliriumassociated clinical outcomes will be evaluated, as either data during hospitalisation and follow-up data until 6 months postoperatively, will be collected.

- Due to the nature of the intervention, blinding of the patients and data collectors was not possible, which is a limitation. However, we expect a low risk of bias in the clinical assessment, as the onset of delirium is not considered a subjective outcome.

\section{INTRODUCTION}

Delirium is characterised by an acute and temporary decline in mental status affecting attention, awareness, cognition, language and visuospatial ability. ${ }^{1}$ This decline is caused by dysregulation of neuronal activity secondary to several pathophysiological disturbances. ${ }^{2}$ Surgery within the brain parenchyma evokes an inflammatory reaction resulting in the formation of oedema and decrease of vascular permeability with impaired oxygenation of nearby tissue resulting in the generation of oxidative stress. Hypotheses describing the pathophysiology of delirium rely on neuroinflammatory and oxidative reactions within the brain. ${ }^{3}$ Considering this, it is plausible that neurosurgical patients are in particular vulnerable to developing postoperative delirium and that the incidence of delirium in this population is high.

Incidence rates of postoperative delirium after intracranial surgery vary between $4 \%$ and $44 \%$ depending on the type of surgery, such as major neurovascular reporting higher 
incidence rates and method of delirium assessment, such as short follow-up duration resulting in lower incidence rates. ${ }^{4-13}$

Delirium often causes a traumatic experience for the patient and his or her relatives. Delirium also leads to up to twice the length of hospital stay, twice the intensity of nursing hours, almost twice the amount of reoperations with additional exposure to complications, three times the costs and more than five times higher mortality risk. ${ }^{6714}$ Delirium can cause in the long term a decline in subjective memory, cognitive decline and increase the chance of developing dementia. ${ }^{15-17}$ These observations warrant the search for preventive therapies for postoperative delirium.

Several preventive pharmacological interventions for occurrence of postoperative delirium have been studied. Pharmacological interventions, targeted at the psychotic symptoms such as olanzapine or haloperidol, at the sleepwake cycle such as melatonin, or lowering sedation levels through Bispectral Index (BIS), were either ineffective or non-reproducible in preventing delirium after surgery. ${ }^{18}$ Furthermore, most of these drugs may have severe side effects. ${ }^{19-21}$

Non-pharmacological multicomponent approaches such as the Hospital Elder Life Programme or the Perioperative Optimisation of Senior Health programme are promising, showing a relative reduction of delirium in $36 \%-77 \% .^{22}{ }^{23}$ However, success of these multicomponent strategies is dependent on the adherence while implementation is challenging and not always adjusted to the feasibility for nurse or patients' needs. ${ }^{24}$

Recorded music is effective in reducing preoperative anxiety, postoperative pain and its stress response induced by surgery. Moreover, lower doses of opioids and sedatives are required when music around surgery is applied with the strongest effect of music in case of patients-own choice irrespective of own music or from preselected playlists. ${ }^{25-33}$ These positive effect on a combination of delirium-eliciting factors might contribute to a reduction of postoperative delirium.

Three studies have been published on the effect of music as a sole intervention on the occurrence of postoperative delirium. One is a five-armed trial with a total of 126 patients (approximately 25 per arm) in which no significant effect was seen. However, this study lacked a solid power analysis. ${ }^{34}$ The second trial had no delirium in either the music and control group due to their exclusion criteria and therefore no effect could be demonstrated. ${ }^{35}$ The third trial randomised 22 patients and reported significant better outcome in the music group. ${ }^{36}$ In none of these trials, the music selection was based on patient's preference. In conclusion, although suggestive, currently no strong evidence exists on the possible beneficial effect of music on delirium.

Furthermore, evidence on the effects of music interventions on delirium-related health outcomes such as length of stay, daily functioning, costs, quality of life and costeffectiveness is lacking. This is a significant knowledge gap, as these truly represent clinically relevant outcome measures for patient and society.

Therefore, this article reports on a randomised control trial to assess the effect of music in the prevention of postoperative delirium in neurosurgical patients.

\section{METHODS AND ANALYSIS \\ Study design}

This study is a randomised controlled trial with two study arms, designed to compare the effects on postoperative delirium, of perioperative recorded music intervention in addition to standard care (intervention group) versus standard care (control group)-prior, during and after a craniotomy. Figure 1 shows the flow diagram of the progress through the trial phases of the two study groups. We will include 189 adults at the Neurosurgery department of the Erasmus Medical Center (Erasmus MC) in Rotterdam. Ethical Committee approval was obtained in April 2020, the first patient was included in July 2020 and July 2022 is the anticipated end date of inclusion. This study protocol followed the Standard Protocol Items: Recommendations for Interventional Trials (SPIRIT) guidelines (see SPIRIT checklist in online supplemental material) and the Consolidated Standards of Reporting Trials (CONSORT) guidelines for non-pharmacological treatments.

\section{Randomisation, blinding and treatment allocation}

The random allocation sequence will be computer generated using an online software program or website (ALEA; FormVision, Abcoude, The Netherlands) ran by the executing researcher after obtaining informed consent. Randomisation will be in a 1:1 ratio and stratified per type of disease characteristic (ie, 'neuro-oncology', 'neurovascular', 'traumatic brain injury', 'infectious') and age (ie, 'younger than 60 years', '60 years or older'). Variable block sizes will be used; in each block both groups will be represented equally. The web-based programme will be secured and only members of the study staff will have login credentials.

Patients participating in the study cannot be blinded due to the nature of the treatment. Selective blinding of the clinicians and data collectors is unsecure while patients might report their experience when undergoing the (music/control) intervention. Hence, to prevent misleading conclusions an unblinded design was chosen. In our view, this is not too much of a limitation, since the primary outcome of this study (ie, the onset of delirium) can be assessed objectively.

As the intervention is without risks and cannot be blinded, it will in no case be necessary to break the randomisation code. Data collection and intervention administration (conducted by the treating nurses and consulting psychiatrist) and randomisation and final analysis (conducted by the executing researcher) were separated but not masked from each other. 


\section{Screening and recruitment}

Eligible patients planned for craniotomy at the Erasmus Medical Center in Rotterdam approached at the outpatient clinic or ward.

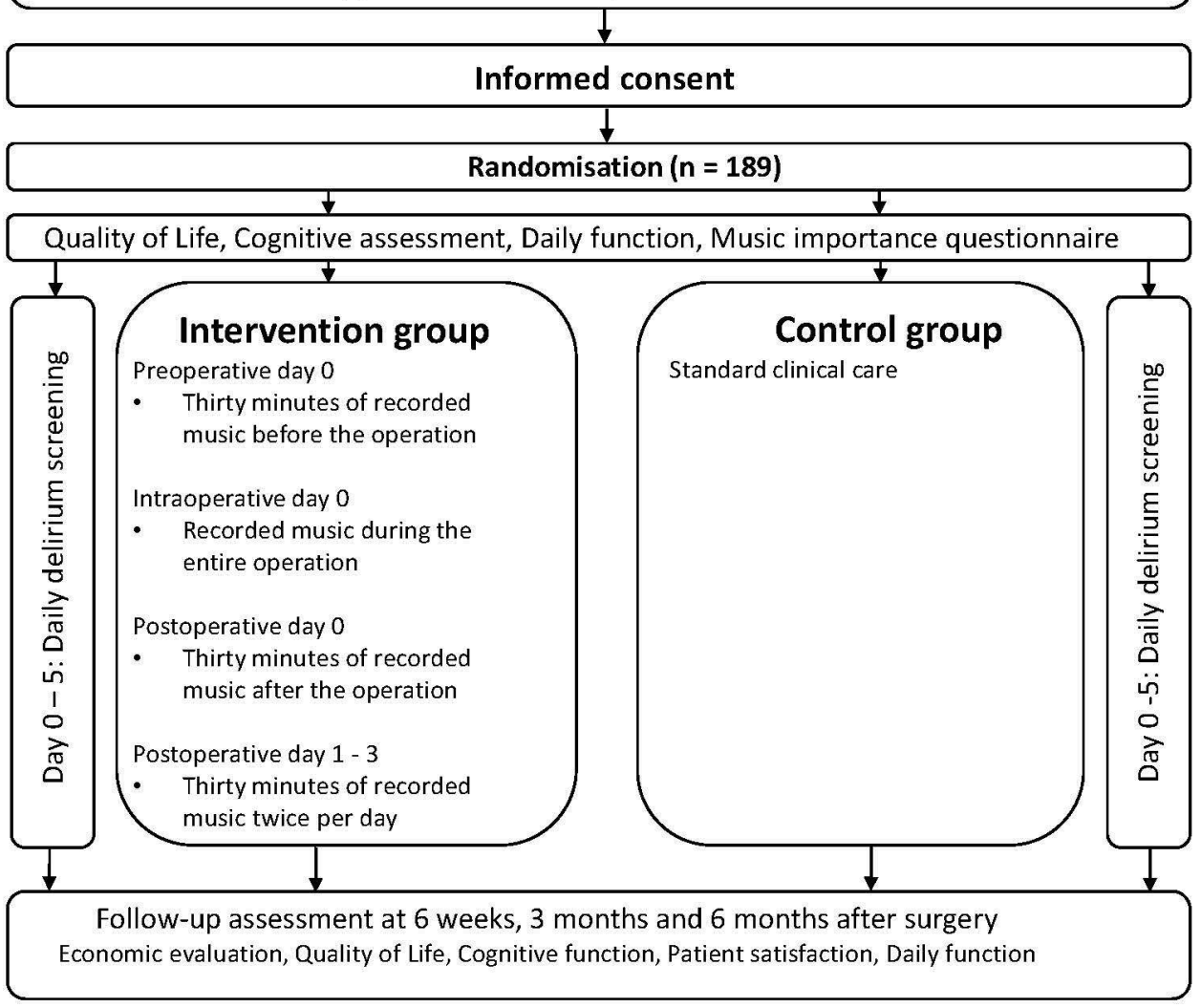

Figure 1 Flow diagram trial phases progress.

\section{Interventions}

Participants in the intervention group (ie, music group) receive an overear headphone and a tablet with access to a platform with different music playlists. These lists are based on personal preference gathered from questionnaires at baseline assessing the role (ie, just listening vs playing instruments), importance (ie, through a Visual Analogue Scale (VAS) from 0 to 100) and preference of music (ie, on genre) per patient. These preselected playlists are categorised based on genre (jazz, blues, classic, electronic, pop, 60s, 70s, 80s, etc), country or artist, are either custom made or composed by our research group from earlier trials and have a minimum duration of 180 min to prevent repetition of songs within the same music session. ${ }^{29}$ The first $30 \mathrm{~min}$ of music, administered by the treating nurse, is given the day of operation with the overear headphones while awaiting surgery. Once in the operating room they will receive in-ear earphones after intubation, compatible with the Mayfield clamp and site of operation. The intraoperative music intervention, in accordance with the preoperative choice of music, will be continued during the surgical procedure and discontinued just before detubation. Although patients might not remember this music session, we chose for music during general anaesthesia as a significant decrease in pain and anxiety has been reported in surgical patients when receiving intra-operative music. ${ }^{33}$ The intraoperative music session is continuous and the duration depends on the duration of surgery and will be documented. After surgery, during recovery at the postoperative care unit another $30 \mathrm{~min}$ of recorded music through overear headphones will be administered. Subsequently, participants will receive $30 \mathrm{~min}$ of recorded music twice a day for the following three postoperative days as music is currently investigated as preventive therapy and onset of postoperative delirium has been reported in the first 3-5 days after intracranial surgery. ${ }^{510} 37-39$

The control group will not receive headphone music and will be treated according to standard care. We did not choose for overear headphone-without music or other auditory signals-in our control group as this is considered an intervention requiring another study arm, which we deemed unfeasible. It would be an interesting opportunity for future research to include other comparison and control groups (exposed to other auditory input or silence), which could also generate more options for blinding the clinicians and data collectors.

All participating subjects in this study will be requested to refrain from listening to music through headphone during the first three postoperative days, apart from the planned intervention. Music other than from the headphone (eg, television) is allowed in either the music or control group but patient or a family member is asked to report this. 
Patients in either group, besides the screening tools for our primary and secondary outcomes, will receive standard clinical care and will not be restricted from any treatments whatsoever.

\section{Outcome parameters}

The primary outcome measure is presence or absence of postoperative delirium within the first five postoperative days. ${ }^{40}$ All participating patients on the ward will be screened daily by the treating nurse using the Delirium Observation Screening (DOS) scale, a validated 13-item delirium screening tool which is already current practice at the Neurosurgical ward in the Erasmus MC. ${ }^{42-46}$ In case of raised suspicion by the DOS a psychiatrist is consulted to confirm or reject clinical diagnosis of delirium based on the Diagnostic and Statistical Manual of Mental Disorders (DSM)-5 criteria. $^{1}$

Secondary outcome parameters include risk factors and health outcomes, which substantiate the effect of music on delirium and evaluate its clinical implications for patient and society:

- Severity and duration of delirium. In case of positive delirium, its severity will be assessed using the Delirium Rating Scale-revised-98. ${ }^{478}$ A DOS score of lower than 3 during 24 hours will be considered as a 'faded out' delirium and number of days from onset until end will be documented.

- Preoperative anxiety assessed with the VAS-anxiety. This 11-point scale, in which 0 implies no anxiety and 10 the worst anxiety possible, is easy to use, highly correlated with the State-Trait Anxiety Inventory, and is assessed while awaiting surgery. In case of visual impairment, caused by the neurological disease, VAS will be exchanged for Numeric Rating Scale (NRS) ${ }^{49-52}$

- Activation of the parasympathetic nervous system, before and after surgery, using the heart rate variability (HRV). The HRV, the variation in the time interval between adjacent heartbeats related to parasympathetic influences, is measured through ECG recordings while awaiting and when recovering from surgery. ${ }^{53}$

- Depth of anaesthesia is registered with BIS, which signals Electroencephalography (EEG) brain activity displayed into numerical values. The BIS is often used to guide during anaesthesia but its feasibility and implications during neurosurgical operations is still unknown..$^{54}$

- Perioperative medication use, such as opioids, benzodiazepines and antipsychotic drugs, will be extracted from the electronic patient files.

- Postoperative pain, assessed using the validated 11-point NRS-scale, in which 0 implies no pain and 10 the worst pain possible. ${ }^{56}$

- Postoperative complications defined as an adverse event within 2 weeks after surgery resulting in prolongation of current admission, new treatment (ie, reoperations) or death.
- Hospital length of stay in days defined as the day of admission until the actual day of discharge.

- Cognitive function assessed with the Montreal Cognitive Assessment tool at baseline, 3 and 6 months. ${ }^{57}$

- Daily function expressed in Karnofsky Performance Scale and modified Ranking Scale. ${ }^{58-60}$ This is assessed at baseline, 6 weeks, 3 and 6 months after surgery.

- Mortality and readmission rate will be evaluated during the follow-up at 6 weeks, 3 and 6 months.

- Health-related quality of life with the European Organisation For Research And Treatment Of Cancer (EORTC)-C30 and the EORTC-BN20 questionnaires at baseline and during the follow-up at 6 weeks, 3 and 6 months.

- Music importance (ie, based on a VAS in which 0 implies no importance at all and 100 the most imaginable importance), preference (ie, chosen per genre) and the role of music (ie, just listening/active playing) is administered at baseline. Moreover patient satisfaction, whether patient received music or not, is assessed at 6 weeks after discharge. ${ }^{61}$

- Economic evaluation; see below for further details.

\section{Eligibility criteria}

Potential subjects visiting the outpatient clinic or admitted to the neurosurgical ward will be informed about our study. A member of the research team undertakes the initial screening for eligibility. In order to be eligible to participate in this study, a subject must meet all of the following inclusion criteria:

- Patients undergoing a craniotomy.

- Adult patients (ie, age 18 years or more).

- Sufficient knowledge of the Dutch language to understand the study documents in the judgement of the attending physician or researcher.

- Provision of written informed consent by patient or legal representative.

A potential subject who meets any of the following criteria will be excluded from participation in this study:

- Impaired awareness before surgery (ie, motoric less than 6 in the Glasgow Coma Scale).

- Planned postoperative ICU admission (ie, with prolonged sedation and mechanical ventilation).

- Suspected delirium (defined as fluctuating awareness) before surgery.

- Current antipsychotic treatment.

- Patients undergoing surgery impeding supply of music (eg, surgical translabyrinthine approach, awake surgery).

- Severe bilateral hearing impairment, defined as no verbal communication possible.

- Known musicogenic epilepsy (ie, seizure provoked when hearing a specific type of sound or musical stimuli).

- Current participation in other clinical trials interfering with results. 


\section{Sample size}

We expect an incidence of delirium in our control group of $30 \%$. This is based on literature documenting incidence of delirium in neurosurgical patients in a northern European population of 29\%-33\% ${ }^{4-68-1362}$ The expected effect cannot be based on previous literature since no adequate trials exist on the effect of music on delirium. Other non-pharmacological interventions mention a relative reduction of $36 \%-77 \% .{ }^{192}$ We will, therefore, consider the intervention clinically relevant if a relative reduction of $60 \%$, corresponding to an absolute reduction of $18 \%$, is achieved. Assuming a power of $80 \%$, a twosided $p$ value of 0.05 , and 1:1 randomisation, a sample size of 90 patients per arm would be required. We expect a lost to follow-up of $5 \%$ and will, therefore, include 189 patients.

\section{Inclusion period}

We expect $50 \%$ of the craniotomy patients not to be eligible due to inclusion or exclusion criteria given above. This leaves 240 eligible patients each year, taking into account that approximately 480 craniotomies are conducted at the Erasmus MC in Rotterdam each year. In $30 \%$ of these cases, it concerns emergency operations and we do not expect to be able to include many of these patients. Considering this, we would in theory therefore need 14 months for inclusion. Hence, we would plan 24 months of inclusion time taking into account all the logistic challenges. In practice, this comes down to one or two inclusions each week.

\section{Statistical analysis}

All analyses will be conducted according the intention-totreat principle, that is, patients will be analysed according to the treatment arm they were assigned to, irrespective of the treatment they actually received. The primary endpoint in a patient will be the occurrence of a DOS score 3 or higher subsequently confirmed with the DSM-5 by a psychiatrist. Those patients will be considered as event, all other patients will be considered as non-event. The proportion of patients with an event will be compared between the randomisation arms using univariate and multivariate logistic regression analysis, that is, the OR with $95 \%$ CI will be calculated. A two-sided $p$ value of 0.05 or less will be considered statistically significant. All other analyses will be exploratory and therefore as hypothesisgenerating only.

\section{Economic evaluation}

Taking a societal perspective, we will analyse the cost-effectiveness of the music intervention versus 'standard care', using the techniques of a trial-based costeffectiveness analysis and cost-utility analysis. Established methods for economic evaluations in healthcare will be used. ${ }^{63-65}$

The analysis will include both medical and non-medical costs. Medical costs include all the costs of hospital admissions, surgeries, diagnostic imaging, laboratory findings and consultations. The cost analysis will include costs of treating adverse consequences of delirium (such as falls and post-traumatic stress) and will extend beyond the initial hospital admission, including visits to the outpatient department, readmissions, nursing home admissions, medications and consultations with psychiatrists. To collect data on healthcare use, both the hospital's electronic information system and data from the iMTA Medical Consumption Questionnaire (administered to the patients at the follow-up visits) will be used. ${ }^{66}$ These data will then be combined with unit costs to generate patient-level costs. Non-medical costs will comprise costs of lost productivity. After all, it is expected that patients in the intervention group may resume their (paid and/ or unpaid) work earlier, as the occurrence of delirium declines. Productivity losses will be measured and valued using the iMTA Productivity Cost Questionnaire ${ }^{67}$ Finally, for the patients in the intervention group, the costs of the music intervention itself (ie, headphones, earphones and sound equipment) will be added.

To measure the effects of the intervention, the economic evaluation will consider the occurrence of delirium (as defined above) and quality-adjusted life-years (QALYs). The calculation of QALYs will be based on survival data and on the EuroQol (EQ)-5D questionnaire. ${ }^{68}$ The EQ-5D is a generic, preference-based quality of life measure, comprising five dimensions of health, that allows for the calculation of QALYs. The EQ-5D will be administered at base line and at 6 weeks and 3 and 6 months follow-up.

Then, incremental cost-effectiveness ratios (ICERs) will be calculated by dividing the difference in costs between the groups by the difference in effects, unless one treatment dominates the other (ie, has lower costs and greater effects). The ICERs will be expressed as incremental costs per case of delirium prevented and incremental costs per QALY gained. Uncertainty in the estimation of the ICERs will be illustrated through cost-effectiveness planes (via bootstrapping). Cost-effectiveness acceptability curves will be calculated showing the probability of the intervention being cost-effective compared with 'standard care' as a function of society's willingness-to-pay for a QALY gained. The time horizon of the analysis will be the 6 months follow-up period. As a result, discounting of future costs and benefits will not be required. Sensitivity analysis will be performed to assess the robustness of the analysis to certain assumptions.

\section{Patient and public involvement}

Patients are involved in the composition of the music playlists, as these are based on their music preference, the role music plays in their life (ie, whether they are musician/just listen to music) and the importance of music. The results of our trial will be disseminated to the participating patients through a letter after publication.

\section{Trial monitoring}

Based on the small chance of damage due to the intervention, our risk is expected to be negligible (risk class 
A). Monitoring will be conducted for quality assurance of data, patient inflow, meeting of inclusion and exclusion criteria, informed consent, compliance, patient safety, study procedures and source document verification in compliance with the monitoring plan for risk class A (negligible risk).

Our monitor will be an independent qualified researcher who completed a Good Clinical Practice training course. Results, conclusion and advice will be recorded in the monitor report and stored for at least 15 years.

All investigators and study staff will be responsible for reporting adverse effects to the coordinating investigator. The coordinating investigator or principal investigator will report adverse events to the Medical Ethics Review Board in accordance with the ethics committee adverse event reporting procedures. The coordinating investigator and the principal investigator are responsible for adherence to all ethical committee rules and guidelines and for the accuracy and completeness of all forms, entries and informed consent.

\section{Data management}

Data will be handled confidentially in compliance with the EU General Data Protection Regulation and the Dutch Act on Implementation of the General Data Protection Regulation (Dutch: Uitvoeringswet Algemene Verordening Gegevensbescherming). Each subject will receive an identification (ID) code which will be based on a random number produced by the randomisation software ALEA and the database tracing towards the patients' ID will be stored separately. Any information on paper collected during this study will be placed in a research folder, which will be filed in locked cabinets in research offices at the Erasmus MC. Any electronic information acquired during the research period will be stored in Open Clinica, a secured and Erasmus MC approved storage programme which tracks all the changes applied and freezes data when inclusion and data check has been done. Only the study staff will have access to the research data.

\section{ETHICS AND DISSEMINATION \\ Ethics}

The study protocol has been reviewed by the Medical Ethics Review Board of the Erasmus MC in Rotterdam on 9 March 2020 and is not subject to the Medical Research Involving Human Subjects Act (Dutch: Wet medischwetenschappelijk onderzoek met mensen / WMO). This study is being conducted according to the principles of the Declaration of Helsinki (64th WMA General Assembly, Fortaleza, Brazil, October 2013).

\section{Benefits and risks assessment}

Listening to music might be experienced as pleasant. During the informed consent process, it will be made clear that participation might not have clear direct benefits to the patient, and that refusal to participate will not have impact on the care received by any of the medical staff.

Recent meta-analysis showed no side effects of recorded music through headphones. ${ }^{28}$ Hypothetically there is a chance of hearing damage-with subsequent tinnituswhich will be minimalised by setting a volume limit of $60 \mathrm{~dB}$ on each tablet, which is the advised loudness of a music intervention in medical care. ${ }^{69}$ Moreover, participants might be upset of being refrained from music when allocated in the control group. Lastly, communicating at the clinician might be complicated during the music session, especially in immobile patients.

All adverse events will be documented. We expect no intervention-related serious adverse events.

\section{Dissemination}

The research team is committed to full disclosure of the results of the trial. Findings will be reported in accordance with CONSORT guidelines and we aim to publish in high-impact journals. Given the multitude of outcome parameters, results will be divided over several papers. The funder will take no role in the analysis or interpretation of results.

\section{Author affiliations}

${ }^{1}$ Neurosurgery, Erasmus Medical Center, Rotterdam, The Netherlands

${ }^{2}$ Neuroscience, Erasmus MC, Rotterdam, The Netherlands

${ }^{3}$ Surgery, Erasmus MC, Rotterdam, The Netherlands

${ }^{4}$ Anesthesiology, Erasmus MC, Rotterdam, The Netherlands

${ }^{5}$ Department of Psychiatry, Erasmus Medical Center, Rotterdam, The Netherlands

${ }^{6}$ Pediatric Surgery, Erasmus MC Sophia, Rotterdam, The Netherlands

${ }^{7}$ Medical Technology Assessment (iMTA), Erasmus Universiteit Rotterdam, Rotterdam, The Netherlands

Correction notice This article has been corrected since it was first published. The middle name for the author SA Kushner and PR Kappen has been added.

Contributors Each author has contributed significantly to, and is willing to take public responsibility for, one or more aspects of the study. AJPEV, CMFD, JJ and PK conceived the study idea. PK coordinated the research protocol and wrote the first draft of the manuscript. JJ, CMFD, MK, SAK, R-JO, MC, MJP and AJPEV critically revised the manuscript. All authors have seen and approved the final version of the manuscript being submitted. The article is the authors' original work, has not received prior publication and is not under consideration for publication elsewhere.

Funding This research is funded with the Erasmus MC Efficiency Grant (Grant number: 19105) by the Erasmus Medical Centre, Rotterdam, The Netherlands.

Competing interests None declared.

Patient consent for publication Not applicable.

Provenance and peer review Not commissioned; externally peer reviewed.

Supplemental material This content has been supplied by the author(s). It has not been vetted by BMJ Publishing Group Limited (BMJ) and may not have been peer-reviewed. Any opinions or recommendations discussed are solely those of the author(s) and are not endorsed by BMJ. BMJ disclaims all liability and responsibility arising from any reliance placed on the content. Where the content includes any translated material, BMJ does not warrant the accuracy and reliability of the translations (including but not limited to local regulations, clinical guidelines, terminology, drug names and drug dosages), and is not responsible for any error and/or omissions arising from translation and adaptation or otherwise.

Open access This is an open access article distributed in accordance with the Creative Commons Attribution Non Commercial (CC BY-NC 4.0) license, which permits others to distribute, remix, adapt, build upon this work non-commercially, and license their derivative works on different terms, provided the original work is properly cited, appropriate credit is given, any changes made indicated, and the use is non-commercial. See: http://creativecommons.org/licenses/by-nc/4.0/. 
ORCID iDs

Pablo R Kappen http://orcid.org/0000-0003-4987-297X

M Klimek http://orcid.org/0000-0002-0122-9929

\section{REFERENCES}

1 American Psychiatric A. American psychiatric association DSM5 Task force (2013): diagnostic and statistical manual of mental disorders: DSM-5. Washington, DC: American Psychiatric Association, 2013.

2 Maldonado JR. Acute brain failure: pathophysiology, diagnosis, management, and sequelae of delirium. Crit Care Clin 2017;33:461-519.

3 Maldonado JR. Neuropathogenesis of delirium: review of current etiologic theories and common pathways. Am J Geriatr Psychiatry 2013;21:1190-222.

4 Matano F, Mizunari T, Yamada K, et al. Environmental and clinical risk factors for delirium in a neurosurgical center: a prospective study. World Neurosurg 2017;103:424-30.

5 Wang J, Ji Y, Wang N, et al. Risk factors for the incidence of delirium in cerebrovascular patients in a neurosurgery intensive care unit: a prospective study. J Clin Nurs 2018;27:407-15.

6 Zipser CM, Deuel J, Ernst J, et al. The predisposing and precipitating risk factors for delirium in neurosurgery: a prospective cohort study of 949 patients. Acta Neurochir 2019;161:1307-15.

7 Schubert M, Schürch R, Boettger S, Maria Schubert RS, Nuñez $\mathrm{DG}$, et al. A hospital-wide evaluation of delirium prevalence and outcomes in acute care patients - a cohort study. BMC Health Serv Res 2018;18:550.

8 Tanaka M, Tani N, Maruo T, et al. Risk factors for postoperative delirium after deep brain stimulation surgery for Parkinson disease. World Neurosurg 2018;114:e518-23.

9 Chen L, Xu M, Li G-Y, et al. Incidence, risk factors and consequences of emergence agitation in adult patients after elective craniotomy for brain tumor: a prospective cohort study. PLoS One 2014;9:e114239.

$10 \mathrm{He} \mathrm{Z}$, Cheng $\mathrm{H}$, Wu H, et al. Risk factors for postoperative delirium in patients undergoing microvascular decompression. PLoS One 2019;14:e0215374.

11 Flanigan PM, Jahangiri A, Weinstein D, et al. Postoperative delirium in glioblastoma patients: risk factors and prognostic implications. Neurosurgery 2018;83:1161-72.

12 Budènas A, Tamašauskas Šarūnas, Šliaužys A, et al. Incidence and clinical significance of postoperative delirium after brain tumor surgery. Acta Neurochir 2018;160:2327-37.

13 Morshed RA, Young JS, Safaee M, et al. Delirium risk factors and associated outcomes in a neurosurgical cohort: a case-control study. World Neurosurg 2019;126:e930-6.

14 Leslie DL, Marcantonio ER, Zhang Y, et al. One-Year health care costs associated with delirium in the elderly population. Arch Intern Med 2008;168:27-32.

15 Kat MG, Vreeswijk R, de Jonghe JFM, et al. Long-Term cognitive outcome of delirium in elderly hip surgery patients. A prospective matched controlled study over two and a half years. Dement Geriatr Cogn Disord 2008;26:1-8.

16 Bickel H, Gradinger R, Kochs E, et al. High risk of cognitive and functional decline after postoperative delirium. A three-year prospective study. Dement Geriatr Cogn Disord 2008;26:26-31.

17 Rockwood K, Cosway S, Carver D, et al. The risk of dementia and death after delirium. Age Ageing 1999;28:551-6.

18 Siddiqi N, Harrison JK, Clegg A, et al. Interventions for preventing delirium in hospitalised non-ICU patients. Cochrane Database Syst Rev 2016;3:CD005563.

19 Janssen TL, Alberts AR, Hooft L, et al. Prevention of postoperative delirium in elderly patients planned for elective surgery: systematic review and meta-analysis. Clin Interv Aging 2019;14:1095-117.

20 Liu Y, Li X-J, Liang Y, et al. Pharmacological prevention of postoperative delirium: a systematic review and meta-analysis of randomized controlled trials. Evid Based Complement Alternat Med 2019;2019:9607129:1-10.

21 Vlisides P, Avidan M. Recent advances in preventing and managing postoperative delirium. F1000Res 2019;8. doi:10.12688/ f1000research.16780.1. [Epub ahead of print: 0105 2019].

22 Abraha I, Trotta F, Rimland JM, et al. Efficacy of non-pharmacological interventions to prevent and treat delirium in older patients: a systematic overview. The SENATOR project ONTOP series. PLOS One 2015;10:e0123090.

23 Hshieh TT, Yang T, Gartaganis SL, et al. Hospital elder life program: systematic review and meta-analysis of effectiveness. Am J Geriatr Psychiatry 2018;26:1015-33.
24 Inouye SK, Westendorp RGJ, Saczynski JS. Delirium in elderly people. Lancet 2014;383:911-22.

25 Cerejeira J, Batista P, Nogueira V, et al. The stress response to surgery and postoperative delirium: evidence of hypothalamicpituitary-adrenal axis hyperresponsiveness and decreased suppression of the GH/IGF-1 axis. J Geriatr Psychiatry Neurol 2013;26:185-94.

26 Clegg A, Young JB. Which medications to avoid in people at risk of delirium: a systematic review. Age Ageing 2011;40:23-9.

27 Fu VX, Oomens P, Sneiders D, et al. The effect of perioperative music on the stress response to surgery: a meta-analysis. J Surg Res 2019;244:444-55.

28 Fu VX, Oomens P, Klimek M, Verhofstad MHJ, et al. The effect of perioperative music on medication requirement and hospital length of stay: a meta-analysis. Ann Surg 2020;272:961-72.

29 Kühlmann AYR, de Rooij A, Kroese LF, et al. Meta-Analysis evaluating music interventions for anxiety and pain in surgery. Br J Surg 2018;105:773-83.

30 Lee JH. The effects of music on pain: a meta-analysis. J Music Ther 2016;53:430-77

31 Leung JM, Sands LP, Paul S, et al. Does postoperative delirium limit the use of patient-controlled analgesia in older surgical patients? Anesthesiology 2009;111:625-31.

32 Schor JD, Levkoff SE, Lipsitz LA, et al. Risk factors for delirium in hospitalized elderly. JAMA 1992;267:827-31.

33 Hole J, Hirsch M, Ball E, et al. Music as an aid for postoperative recovery in adults: a systematic review and meta-analysis. Lancet 2015;386:1659-71.

34 Iblher $\mathrm{P}$, Mahler $\mathrm{H}$, Heinze $\mathrm{H}$, et al. Does music harm patients after cardiac surgery? A randomized, controlled study. Applied Cardiopulmonary Pathophysiology 2011;15:14-23.

35 Johnson K, Fleury J, McClain D. Music intervention to prevent delirium among older patients admitted to a trauma intensive care unit and a trauma orthopaedic unit. Intensive Crit Care Nurs 2018;47:7-14.

36 McCaffrey $R$. The effect of music on acute confusion in older adults after hip or knee surgery. Appl Nurs Res 2009;22:107-12.

37 Hosoya R, Sato Y, Ishida E, et al. Association between delirium and prehospitalization medication in poststroke patients. J Stroke Cerebrovasc Dis 2018;27:1914-20.

38 Mokhtari M, Farasatinasab M, Jafarpour Machian M, et al. Aripiprazole for prevention of delirium in the neurosurgical intensive care unit: a double-blind, randomized, placebo-controlled study. Eur J Clin Pharmacol 2020;76:491-499.

39 Oh Y-S, Kim D-W, Chun H-J, et al. Incidence and risk factors of acute postoperative delirium in geriatric neurosurgical patients. J Korean Neurosurg Soc 2008;43:143-8.

40 Robinson TN, Raeburn CD, Tran ZV, et al. Postoperative delirium in the elderly: risk factors and outcomes. Ann Surg 2009;249:173-8.

41 Lee H, Ju J-W, Oh S-Y, et al. Impact of timing and duration of postoperative delirium: a retrospective observational study. Surgery 2018;164:137-43.

42 Association AP. Diagnostic and statistical manual of mental disorders. Fourth Edition, 2013.

43 Gavinski K, Carnahan R, Weckmann M. Validation of the delirium observation screening scale in a hospitalized older population. $J$ Hosp Med 2016;11:494-7.

44 Detroyer E, Clement PM, Baeten N, et al. Detection of delirium in palliative care unit patients: a prospective descriptive study of the delirium observation screening scale administered by bedside nurses. Palliat Med 2014;28:79-86.

45 Schuurmans MJ, Shortridge-Baggett LM, Duursma SA. The delirium observation screening scale: a screening instrument for delirium. Res Theory Nurs Pract 2003;17:31-50.

46 van Velthuijsen EL, Zwakhalen SMG, Warnier RMJ, et al. Psychometric properties and feasibility of instruments for the detection of delirium in older hospitalized patients: a systematic review. Int J Geriatr Psychiatry 2016;31:974-89.

47 Grover S, Kate N. Assessment scales for delirium: a review. World J Psychiatry 2012;2:58-70.

48 Trzepacz PT, Mittal D, Torres R, et al. Validation of the delirium rating Scale-revised-98: comparison with the delirium rating scale and the cognitive test for delirium. J Neuropsychiatry Clin Neurosci 2001;13:229-42.

49 Spielberger CD GR, Lushene R, Vagg PR, et al. Manual for the state trait anxiety inventory. Consulting Psychologists Press, 1983.

50 Julian LJ. Measures of anxiety: State-Trait anxiety inventory (STAI), Beck anxiety inventory (BAI), and hospital anxiety and depression Scale-Anxiety (HADS-A). Arthritis Care Res 2011;63 Supp 11:S467-72. 
51 Abend R, Dan O, Maoz K, et al. Reliability, validity and sensitivity of a computerized visual analog scale measuring state anxiety. J Behav Ther Exp Psychiatry 2014;45:447-53.

52 Facco E, Stellini E, Bacci C, et al. Validation of visual analogue scale for anxiety (VAS-A) in preanesthesia evaluation. Minerva Anestesiol 2013;79:R02Y9999NOOA0660:1389-95.

53 Camm AJ, Malik M, Bigger JT. Heart rate variability: standards of measurement, physiological interpretation and clinical use. Task force of the European Society of cardiology and the North American Society of pacing and electrophysiology. Circulation 1996;93:1043-65.

54 Chiu CL, Ong G, Majid AA. Impact of bispectral index monitoring on propofol administration in patients undergoing cardiopulmonary bypass. Anaesth Intensive Care 2007;35:342-7.

55 Gan TJ, Glass PS, Windsor A, et al. Bispectral index monitoring allows faster emergence and improved recovery from propofol, alfentanil, and nitrous oxide anesthesia. bis utility Study Group. Anesthesiology 1997;87:808-15.

56 Chang $\mathrm{C}-\mathrm{H}$, Chang $\mathrm{C}-\mathrm{H}$, Pickard AS, et al. Visual analog scales for assessing surgical pain. J Am Coll Surg 2005;201:S10727515(05)00396-0 [pii]:245-52.

57 Nasreddine ZS, Phillips NA, Bédirian V, et al. The Montreal cognitive assessment, MoCA: a brief screening tool for mild cognitive impairment. J Am Geriatr Soc 2005;53:695-9.

58 Verger E, Salamero M, Conill C. Can Karnofsky performance status be transformed to the eastern cooperative Oncology Group scoring scale and vice versa? Eur J Cancer 1992;28A:1328-30.

59 van Swieten JC, Koudstaal PJ, Visser MC, et al. Interobserver agreement for the assessment of handicap in stroke patients. Stroke 1988;19:604-7.
60 Banks JL, Marotta CA. Outcomes validity and reliability of the modified Rankin scale: implications for stroke clinical trials: a literature review and synthesis. Stroke 2007;38:1091-6.

61 Cruise CJ, Chung F, Yogendran S, Little C, et al. Music increases satisfaction in elderly outpatients undergoing cataract surgery. Can $J$ Anaesth 1997;44:43-8.

62 van den Boogaard M, Schoonhoven L, Evers AWM, et al. Delirium in critically ill patients: impact on long-term health-related quality of life and cognitive functioning. Crit Care Med 2012;40:112-8.

63 Institue NHC. Manual for costing research. Methods and reference prices for economic evaluations in health care. Updated edition [in Dutch. Diemen: National Health Care Institute, 2015.

64 Institute NHC. Guidelines for Economic Evaluations of Health Care [in Dutch]. Diemen: National Health Care Institute, 2015.

65 Sanders GD, Neumann PJ, Basu A, et al. Recommendations for conduct, methodological practices, and reporting of costeffectiveness analyses: second panel on cost-effectiveness in health and medicine. JAMA 2016;316:1093-103.

66 Bouwmans C H-VRL, Koopmanschap M, Krol M, et al. iMTA medical consumption questionnaire. Rotterdam: Institute for Medical Technology Assessment, Erasmus Universiteit Rotterdam, 2013.

67 Bouwmans C, Krol M, Severens $\mathrm{H}$, et al. The iMTA productivity cost questionnaire: a standardized instrument for measuring and Valuing health-related productivity losses. Value Health 2015;18:753-8.

68 Brooks R. EuroQol: the current state of play. Health Policy 1996;37:53-72.

69 Nilsson $U$. The anxiety- and pain-reducing effects of music interventions: a systematic review. Aorn J 2008;87:S00012092(07)00575-3 [pii]:780-807. 